contraction and was divided by numerous fibrous septa stretching across it, separating it into smooth-walled loculi, which communicated with each other. The left lung was much enlarged and congested, the edges being highly emphysematous. It contained cheesy nodules, around which were miliary tubercles of recent date. In the portion drawn over to the right side was a cavity of the size of a walnut, which corresponded to the area of the cavernous respiration heard under the right clavicle. The heart was large, the right ventricle was slightly dilated, and the valves were healthy; the liver and spleen were both lardaceous; the kidneys were healthy.

In this case the shrinking of the right lung had caused remarkable changes. The chest wall was contracted, the liver pressed upwards, the heart was displaced to the right of the sternum, and the upper part of the left lung into the right chest. Owing to the portion of the left lung, which had been drawn over, containing a cavity, it gave rise to cavernous sounds under the right clavicle, from which it was naturally concluded that the cavity existed in the right lung, whereas, strange to say, it was in the left lung but in the right chest. It is probable that Sir R. Douglas Powell and Dr. Cotton listened to a different cavity from the one I detected, as their examination of the patient's chest was one and a half years before mine, and great changes intervened in that period, the right lung contracting steadily. Death was caused probably by the eruption of miliary tubercle in the left lung, by which the already limited respiratory area was still further reduced.

In concluston, Gentlemen, the above facts show that though fibrosis is the process by which Nature resists the advance of tuberculosis, and limits its inroads, its progress and spread in the tissues are by no means an unmixed blessing to the patient, as it often obstructs the circulation, causing dropsy, and goes nigh to strangle him by reducing still further his already limited breathing surface.

\section{SOME FURTHER INVESTIGATIONS AND OBSERVATIONS UPON THE PATHO- \\ LOGY OF RHEUMATIC FEVER.}

By F. J. POYNTON, M.D. LoND., F.R.C.P. LoND.,

HONORARY PHYSTCTAN TO ST. SAVIOUR'S HOSPITAL, IONDON; ASSISTAN' PrIYSICIAN TO THE HOSPITAX FOR SICK CHILDREN, GREAT ORMOND-STREET, AND TO UNIVERSITY COLLEGE HOSPITAX

AND

ALEXANDER PAINE, M.D. LOND.,

DIRECTOR OF THE CANCER RESEARCH AND PATHOLOGICAL DEPARTMENT OF THE CANCER HOSPITAL, BROMPTON, S.W.; LECTURER ON BACTERIOLOGY AT THE BEDFORD COILEGE FOR WOMEN, LONDON.

(From the Research Department of University College Hospital Medical Sehool.)

IT is now close upon ten years since we published in THE LANCET ${ }^{1}$ the first evidence that we had at our disposal upon the pathology of acute rheumatism, and while bringing forward some additional points we would shortly review the position as it seems to us to stand to-day.

In our first paper we attempted to show the relation of our own investigations to those of others, and with this we need not deal again here; in our later papers we also referred to the researches of other workers upon this subject, notably Dr. E. W. Ainley Walker, ${ }^{2}$ Dr. W. Vernon Shaw, ${ }^{3}$ and Professor J. M. Beattie. ${ }^{4}$ Much interesting criticism has been directed against the two main conclusions we have so often stated, and which we venture to repeat here: first, that a diplococcus, streptococcus, or micrococcus is a cause and most probably the only exciting cause of acute rheumatism; secondly, that acute rheumatism may be a cause of simple and malignant endocarditis. We will at once clear the ground by adding that we are as convinced now as we were before that both these conclusions hold good. Some of the criticisms that have been put forward have been already

1 The Lancet, Sept. 22nd and 29th, 1900. 2 Ainley Walker: Practitioner, 1903.

3 Vernon Shaw : Journal of Pathology and Bacteriology, 1903.

4 Beattie : Brit. Med. Jour., December, 1904; Journal of Experimental Medicine, wol. ix., No 2, 1907. answered, and in this paper we shall attempt to deal further with one of the most recent of them.

It has been suggested that we have been describing an agonal or terminal infection, but we have brought forward conclusive evidence to show that some of the patients from whom the micrococcus was isolated lived for many weeks afterwards, and that others were alive and at work some years later. This criticism may then be dismissed once and for all. Again, it has been suggested that the micrococcus may be present in complicated rheumatism as an epiphenomenon, as a cause, that is, of the complications. Having produced experimentally the important lesions of the disease, ${ }^{5}$ we naturally ask in return what is to constitute acute rheumatism when deprived of arthritis, carditis, nodules, choreiform movements, and pleurisy? We hold that there is, as in tuberculosis, only a predisposition left in the sense that there is some vulnerability of the tissues to this infection, and that there is no mysterious disease "rheumatism," which is always associated with secondary infections. The idea that rheumatism produces arthritis, but that a secondary infection produces the endocarditis which is so often associated, may now be looked upon as contrary to all accurate knowledge. Another difficulty has been raised that this micrococcus is not constantly present and that we have even ourselves not always succeeded in isolating it. This is naturally a troublesome question to answer, and it has been made all the more so because this further oriticism has been added, that when, on the one hand, some observers are repeatedly finding this micrococcus, and others of equal reputation are always failing, the success of those who obtain positive results is somewhat suspicious.

If, then, we fail to find the infective agent we must be convicted out of our own mouths, and if we find it we are suspects because others do not succeed. With regard to this micro-organism, all our own investigations go to prove that it is very constant. We have not demonstrated it in every case of acute rheumatism, but that is no proof that it may not be present, and, on the other hand, we have succeeded so repeatedly that we believe that we are in the position to say that if in any case one had unlimited scope and time for investigations the results would be quite constant. It is abundantly clear that we must choose the cases with care, and with a knowledge of pathological processes, selecting those in which the disease is active. It is a hopeless undertaking to investigate a case of chronic rheumatic heart disease which has been in extremis from secondary cardiac failure of mechanical origin, the result of the scars of former disease. Again, from one's knowledge of rheumatism, it is hardly likely that 10 cubic centimetres of blood taken at random from the general circulation is going to yield a positive result in a mild rheumatic arthritis.

The particular points that we wish to lay stress on now with reference to the isolation of this micrococcus are that we have upon several occasions demonstrated it in fatal rheumatism and that it has been simultaneously found by others in the same case; secondly, that we have worked with it when isolated not by ourselves but by others; and lastly, that we have isolated it from pericardial fluids obtained from fatal pericarditis by others who have forwarded to us the fluids in sealed tubes. These tubes we have incubated without opening, and as was the case in the first result we ever reported, the diplococcus was found in these fluids after incubation-that is, growing in the natural medium.

Example No. 1.-A girl, aged 7 years, was admitted to University College Hospital on March 25th, 1906, with a three weeks' history of acute malaise, vomiting, and multiple arthritis. Chorea and severe pericarditis developed with endocarditis, and death occurred after barely four weeks' illness. There had been a previous attack of rheumatic fever at five years of age. The post-mortem investigation showed percarditis of the usual rheumatic type.

Dr. F. H. Thiele, clinical pathologist to University College Hospital, obtained a pure growth of a diplococcus from the heart's blood Hospital, Lewis demonstrated a dip diplococeus from the heart's blood. Dr. T. found it in the exudation, and in the laboratory it corformed to the found it in the exudation, and in the laboratory it corformed to the characters of the diplococcus rheumaticus, and on expe

Example No. 2.-In June, 1908, Dr. J. Graham Forbes, bacteriologist to Example No. 2.- In June, 1908, Dr. J. Graham Forbes, bacteriologist to the Hospital for Sick Children, Great Ormond-street, isolated a diplothe blood of a child in the hospital suffering from carditis, chorea, and nodules. This diplo-streptococcus had the usual appearances and growth on cuiture. Dr. Forbes kindly undertaken before-the subcutaneous inoculation of a large dose of the

5 Transactions of the Pathological Society of London, 1901'; Tere LaveET, May 4th, 1901 . 
culture. No suppuration resulted and in a short time all signs of an inflammatory reaction had disappeared as in our previous experience.

It is interesting that the subcultures from this case growing on unsuitable media showed a transition of the diplococcus into bacillary forms. Those who have followed the researches upon Achalme's bacillus will remember that in the last few years it had been observed this bacillus may assume a diplococcal form.

Example No. 3.-Dr. A. Salisbury Macnalty, resident medical officer at the Brompton Hospital for Diseases of the Chest, in April, 1909, kindly sent us a culture of a diplococcus which he had isolated post mortern from a case of rheumatic pericarditis. This micro-organism showed the usual characters of the diplococcus rheumaticus and produced a transitory arthritis of the right knee-joint on intravenous inoculation into a rabbit. Example No. 4.-In August, 1909, through the kindness of Dr.
Lauriston Shaw and Dr. A. F. Hertz from Guy's Hospital, pericardial and cerebro-spinal fluid were taken from a case of fatal rheumatic hyperpyrexia and were forwarded to us in sterilised sealed tubes; the results are fully detailed later in this paper.

We believe that these examples will dispose of any suspicion that our success in isolation of the diplococcus is dependent upon some mysterious chance, and we need only recall the work of Ainley Walker, Vernon Shaw, and Beattie in this country to make it clear that the diplococcus is not a monopoly.

With regard to the specificity of this micro-organism we would again repeat that in our experience no other can be found in the rheumatic lesions in man which will produce similar lesions in animals. Undoubtedly various micrococci may, for example, produce experimental endocarditis, as they may also produce endocarditis in man, but various micrococci are not found in human rheumatic endocarditis which will reprodnce the disease. Again, various infections may canse experimental pericarditis, but only one in our experience can produce rheumatic pericarditis in man and also experimental pericarditis. We hold, too, that Triboulet, Wassermann, Walker, Vernon Shaw, Beattie, and ourselves have shown that the general characteristics of this streptococcus are as well differentiated as those of other members of this group. It would, nevertheless, be wrong to ignore the recent investigations by Rosenthal and others in France upon Achalme's bacillus, or to hide the fact that they claim that they have proved its causal relation to the disease. We can only repeat that we have failed to demonstrate this bacillus, and would add that even the French observers lay stress upon its pleomorphism and its occurrence in diplococoal form. Thus Rosenthal ${ }^{6}$ mentions the reception of a diplococcus from Belgium which had been isolated from the blood of a case of active rheumatism. This, when transferred to his anaerobic media, developed into the bacillus of Achalme, but in aerobic culture it retained its diplococcal form. Rosenthal and others each year that passes are apparently striving to prove that this diplococcus is the bacillus of Achalme growing in unsuitable media. We, on the other hand, maintain that the diplococcus grows naturally in aerobic media, but may, as we stated eight years ago, when growing in unsuitable media, assume bacillary forms. Whether these bacillary forms are Achalme's bacillus we cannot say.

In this country we believe the tendency has been to mystify the main question-the etiology of rheumatismby introducing a classification of the streptococci by certain sugar ferment tests in the laboratory. Apparently, as an outcome of this, we have to be satisfied with the description of a streptococcus salivarius or frealis. That a streptococcus in the saliva or fæces should give certain tests in the laboratory is not likely to be of any practical moment unless some pecaliar streptococci develop de novo in these situations. Seeing that the nature of this group of micro-organisms is much altered by the media in which they grow, such a classification is in our present state of knowledge most perplexing. Instance, for example, the diplococcus of rhenmatism: it undoubtedly locates itself in the tonsils, but are not these washed by the saliva, and is not the saliva swallowed into the stomach and passed into the intestines with the food? Which is of the greater importance-that a diplococcus can be isolated from a rheumatic angina and reproduce rheumatic lesions, or that a diplococcus isolated from the saliva and fæces can give some apparently specific sugar tests in a laboratory? Lastly, what alterations in character and virulence might not the diplococcus undergo when, dislodged from the tonsils by the saliva, it lingers in a new environment such as the fæces?
Is it not an outcome of the contusion which arises from such a classification that in a valuable paper on infective endocarditis we read as follows: "It would obviously be fallacious to conclude that any micro-organism isolated from the blood of a patient with fever, endocarditis, and chorea had any necessary connexion with the cause of rheumatic fever" ? Seeing that a streptococcus isolated from acute rheumatic lesions, and let us add also from the blood in acute rheumatism, can produce endocarditis, fever, and choreiform movements, as more than one skilled bacteriologist has shown, it is difficult to see how the conclusion that this streptococcus has some necessary connexion with rheumatic fever can obviously be fallacious. It may be a wrong conclusion but it seems almost a reasonable one.

\section{Indirect Support from Recent Investigations.}

We would next point out that in the last ten years the trend of scientific investigation and clinical observation has strengthened our position in spite of the fact that some of these observations have been made by those who have not had the causation of rheumatism in view, and others have been made by those who have not accepted our explanation and yet have been of assistance in bringing us support by their independent observations.

To take the first type of evidence, we would allude to a paper written by one of us in 1898 and read at the Royal Medical and Chirurgical Society. ${ }^{7}$ In this contribution it was demonstrated that in rheumatic carditis scattered foci of inflammatory change were to be found in the connective tissue framework of the heart springing from the region of the small blood-vessels. It was further shown that in these regions gross changes might be found in the neighbouring muscular fibres. This distribution, it was pointed out, strongly favoured the view that they were a result of some bacterial infection. Later, when we identified the infection, we produced and showed at the Pathological Sncietr of London experimental carditis of the same order. ${ }^{8}$ The... observations have been expanded, notably by Aschoff and Tawara" and Carey-Coombs, ${ }^{10}$ who have maintained that they are specific lesions, and their importance has been insisted upon from an entirely different standpoint to ours. We emphasised their importance as a factor in the beart failure of rheumatic morbus cordis. Others, led by Dr. James Mackenzie, have dwelt upon their importance in connexion with cardiac arrhythmias and the primitive cardiac muscle. If we recall that Keith, in one of his latest papers which he read upon the anatomical side of this question before the Medical Society of London in 1909, stated his conviction that cardiac nerve and cardiac muscle come into such close relation that they may fairly be said to merge in the primitive muscular fibre of the cardiac tube, it becomes at once apparent that these focal lesions of rheumatic carditis must have an important bearing upon this aspect of cardiac disease.

Since Aschoff published his observations they have attracted considerable attention in this country not because of their bearing upon the etiology of rheumatism, but because they are concerned with the ever fascinating problem of the heart beat.

Next we will take the second type of evidence supplied by others who are opposed to our explanations, but who in spite of this have given us indirect support.

To us there are few more curious points in the history of cardiac pathology than the attitude held by many toward rheumatic endocarditis. They admit it is infective; the evidence, indeed, in its favour is overwhelming, but they hesitate to admit that this endocarditis may become malignant. When, however, we analyse the meaning of this malignancy as applied to endocarditis, we find it expresses an inability on the part of the damaged tissues to overcome the local infection. There may be partial, and exceptionally even complete, success, but the rule is failure. This malignancy is, then, no new mysterious change in the tissues, and as its antecedent we find the most frequent is previous rheumatio endocarditis.

Now come the curious conclusions we are asked to accept: firstly, that an infection in the endocardium of rheumatic origin is always simple-that is, is always capable of

\footnotetext{
7 Foynton: Transactions of the Royal Medical and Chirurgical Society, vol. lxxxii., 1899

Transactions of the Pathological Society of London, 1901; International Clinics, vol. iii... Series 13

Brit. Mi en. Jour., 1906, vol. ii., p. 1103.
}

10 Quarterly Journal of Medicine, vol. ii., No. 5. 
healing; and, secondly, that malignant endocarditis after rheumatism is always caused by a secondary infection of valves scarred by previous disease.

The first conclusion is strange, because infections of the rheumatic type are not likely to be always "simple" ; they obviously vary in virulence as do all other infections of the same group. The second conclusion that bacteria prefer scarred valves is, in our opinion, a false one. There is no evidence that bacteria prefer scar tissue; in fact, all evidence is against it. If the so-called scarred valves of rheumatism are examined it will be found that the vegetations are not really scar tissue, but are masses of unhealthy necrotic material which the scar formation has not invaded. We hold that in this necrotic tissue the rheumatic infection that caused the original endocarditis often lurks quiescent but not destroyed. The formation of scar tissue makes it difficult for leucocytosis to deal with bacteria in these isolated necrotic patches, which, bordering as they do on the general circulation, are as much a source of danger as a necrotic gland close to the circulation is in tuberculosis.

The re zent vegetations of rheumatism are, in our opinion, often much more chronic than is generally believed, and those who investigate them from this point of view will find in them areas of necrotic tissue shut off by scar tissue-that is, they will find imperfect healing. We believe that in cases of malignant endocarditis of rheumatic origin the disease has been slightly active long before symptoms call attention to the serious nature of the disease. This link in the chain of evidence is difficult to get, but doubtless it will be obtained by clinicians who keep a constant watch on their patients who leave hospital with rheumatic heart disease. When in these cases the malignant type arises it is not necessarily because some added infection has attacked scar or sclerotic tissue, but because some agency lowering the general bealth has aroused the lurking micrococci of a previous infection which are lying in the necrotic areas bordering on the general blood stream.

In our original paper ${ }^{11}$ we could not hazard how many cases of malignant endocarditis were rheumatic, but we thought future experience would show that a very considerable percentage would prove to be of this type. We are thus interested to find Dr. T. J. Horder discovering that the type of streptococcus most often found in infective endocarditis is not the highly pathogenic streptococcus of suppurative processes, but what he terms "one or other of three types of streptococci that are more closely allied to the "sapro. phytic 'streptococci of the alimentary canal." Dr. Horder ${ }^{12}$ writes in terms of salivarius and fæcalis which unfortu. nately we cannot follow, but we note also that in an analysis of 100 cases of infective endocarditis he finds a streptococcus was present in 62 We hold that all complete evidence goes to show that the streptococcus which causes acute rheumatism is a cause also of malignant endocarditis, and that the further evidence in this condition of the frequent occurrence of "streptococci," which for want of a better description from others we may call peculiar, is an additional support to this view.

We would add that since our first contribution to this question we have seen further cases in adults and children which confirm our original examples and entirely support the view that rheumatism may be a cause of malignant endocarditis.

Aoute Rhtumatism and Rheumatio Fever Faulty Terms.

The next point we would emphasise is the retarding influence that the terms acute rheumatism and rheumatic fever exert upon the study of rheumatism. If we compare any one other great infection to rheumatism in its clinical course it would be tuberculosis. This is but a repetition of a former statement, but we allude to it now because it is so thoroughly supported by a recent paper by Dr. R. Miller ${ }^{13}$ upon chorea. In this paper the writer dwells upon the fact that the choreiform movements are but the developed disease, and that there may be warnings weeks before these appear. The late Dr. W. B. Cheadle, Sir Thomas Barlow, and Sir Dyce Duckworth have called attention to the same point. This premonitory stage is not peculiar to chorea, for heart disease and arthritis show the same features. Many cases in childhood which develop obvious rheumatism may suffer for

11 Transactions of the Royal Medical and Chirurgical Society, vol. $\operatorname{lx} \times x \times$ v., 1903 .

12 Quarterly Journal of Medicine, April, 1909, vol. ii , No. 7, p. 298. 13 THE LANCET, Dec. 18:h, 1909, p. 1808 , some weeks from fever, anæmia, and debility before anyone can foresee the exact meaning of these symptoms, a point which, we may add, Dr. G. H. M. Dunlop has also insisted upon. How can we, in face of these facts, keep the terms acute rheumatism and rheumatic fever as indicative of anything but phases of rheumatism? We have no doubt that a good deal of the mystery of unexplained heart disease, and in particular mitral stenosis, is the result of this imperfect naming of the disease. It is essential that some more general term such as rheumatism, which implies neither acuteness nor chronicity, should be used.

\section{Rheumatic Hyperpyrexia.}

Hyperpyrexia is an event in rheumatism which all would point to as one which should throw light on the pathology of the disease. Through the kindness of Dr. Lauriston Shaw and Dr. A. F. Hertz we have had assistance from Guy's Hospital upon this question. Until this recent opportunity we had only one case for investigation, and that not a classical example. It was fully published by us in THE LANCET in 1905, ${ }^{14}$ and was peculiar in that there was severe cerebrospinal meningitis. In the exudation there were great numbers of strepto-diplococci, and we satisfied ourselves, but we fear not others, that it was a rheumatic case. There is no necessity to recount the details of that investigation here. Our second from Guy's was of the classical type, the patient, an adult, dying with a temperature of $107^{\circ} \mathrm{F}$., and the postmortem examination showing active rheumatism but no meningitis. The following details give the salient features of this investigation.

The following tubes, among others, were received from Guy's Hospital on August 13th, 1909: (1) the turbid pericardial fluid in a sterilised pipette; (C) the blood; and (3) portions of the cerebral cortex. The two latter were placed in bouillon. The pericardial fluid was incubated overnight without addition of any medium.

The following results were obtained. A pure growth of strepto-diplococci was present in the incubated pericardial fluid (Fig. 1). The cerebral cortex gave a growth of diplo-

FIG. 1.

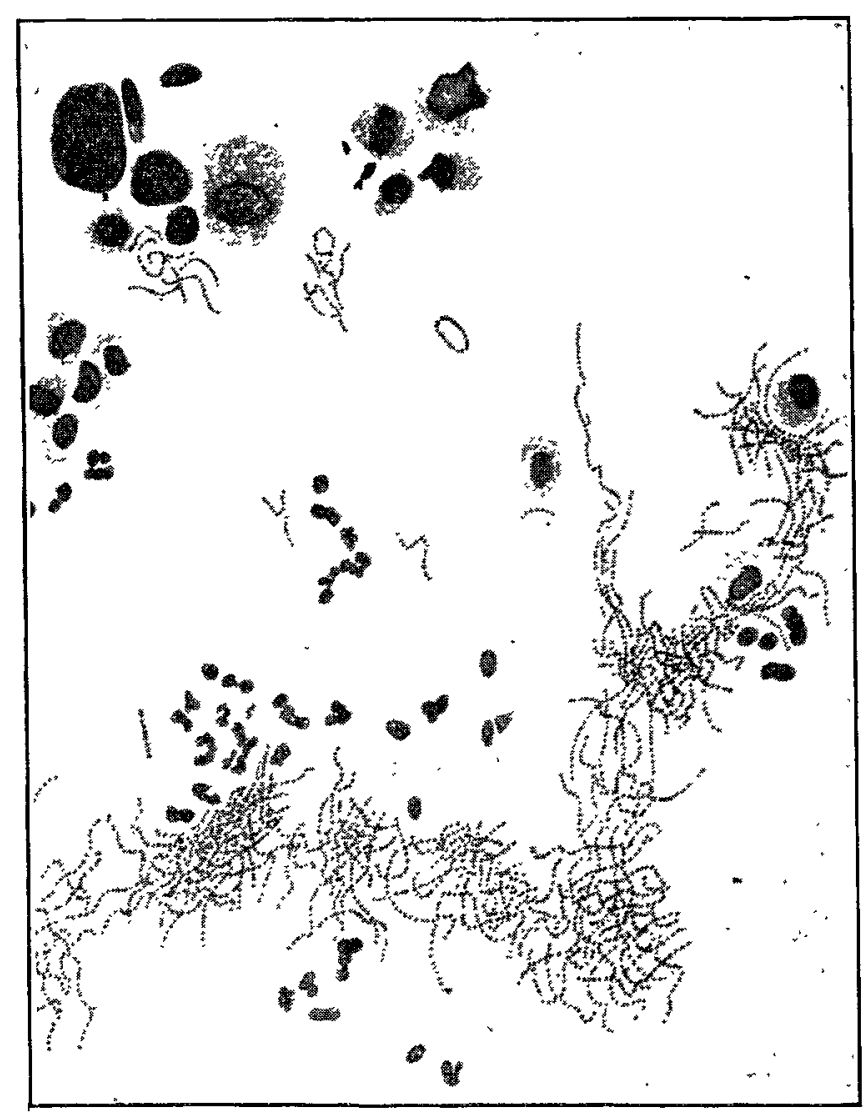

Incubated human pericarial fluid $(x 600)$ from a case of rheumatic pericarditis and fatal hyperpyrexia. Showing polynuclear leucocytes and organisins growing in streptococcal chains

cocci with a few colonies of staphylococcus aureus. The blood gave a growth of diplococci and bacillus coli. A film 
of the cerebro-spinal fluid sbowed a few diplococci. A film of the pericardial fluid showed the diplococci in streptococcal chains ard in considerable number.

On August 15th two intravenous inoculations were made into rabbits. No. 1 received 30 drops of the pericardial fluid. No. 2 received 20 drops of the bouillon culture from the cerebral cortex which contained some colonies of staphylococcus aureus with the diplococcus. The second rabbit died in 24 hours from acute pericarditis, and the pericardial fluid from this, inoculated overnight, showed a pure growth of strepto-diplococci (Fig. 2). The other organs showed no

FrG. 2.

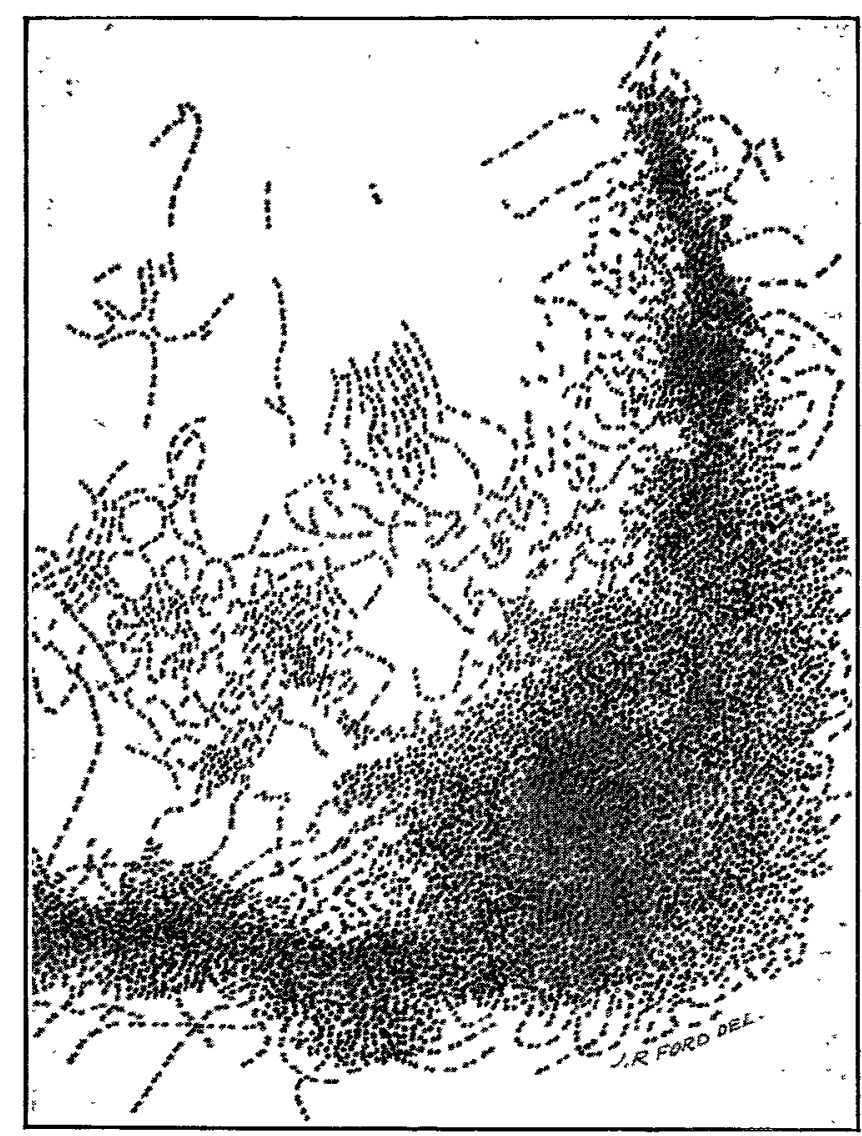

Incubated rabbit pericardial fluid $(x 1000)$. Showing the streptococcal form of the micrococcus growing, as in Fig. 1, in tococcal form of the micrococcus growing, as in Fig. 1, in streptococcal chains. This film is under higher magnificaas well as the micrococci, and required a larger field.

obvious change. They were not soft and there were no hæmorrhages. The first rabbit lived a week and during that time it rapidly wasted and developed pyrexia, endocarditis, and arthritis of the right knee-joint. The post-mortem examination showed early endocarditis of the tricuspid valve, a fibrino-plastic exudation in the right knee-joint, and an infarct in the right kidney. Cultures from the right kneejoint gave a pure culture of strepto-diplococci.

A third rabbit was inoculated intravenously with 10 minims of the incubated pericardial fluid from rabbit No. 1. Three days later there was arthritis of both shoulderjoints with wasting and general illness, but during the next week there was gradual recovery from the arthritis. This animal died from acute intussusception 12 days after the injection, and the post-mortem examination showed no cardiac lesion and a healing arthritis.

A fourth rabbit was inoculated on August 25th with a culture from the cerebro-spinal fluid. On the 28 th the right knee became swollen and tender and the animal began to waste. No cardiac lesion was deterted.

Examination of fragments of the aortic and mitral valves, portions of the motor cortex and pia matex of the patient showed that the valvular lesions were of old standing and that the brain and pia mater examined only showed a few diplococci.

The result of this investigation may be thus summarised : From a case of rheumatic fever with carditis, arthritis, and hyperpyrexia a strepto-diplococcus was obtained in pure culture direct from the pericardial fluid incubated overnight. This strepto-diplococcus produced, on intravenous inoculation into rabbits, non-suppurative multiple arthritis, pericarditis, endocarditis, and infarction, and the micro-organism was again isolated from the lesions in pure culture. The same micro-organism was present in the cerebro-spinal fluid, motor cortex, and blood of the patient.

It would be a mistake to infer too much from a single case, but we believe this investigation supports the general opinion among physicians that rheumatic hyperpyrexia is a peculiar toxæmic process rather than an intense bacterial invasion, for although the micrococci were both isolated and demonstrated, they were not present in the tissues or blood in any extraordinary numbers.

Investigations upon the Tonsils in the Rheumatio.

Two other lines of investigation have occupied our attention in the last five years: the first is another step in the study of the tonsils suggested to us by Mr. George Waugh; the second is an experimental study of arthritis, with a view to ascertaining whether more than one form of arthritis may result from intravenous inoculation of the same micro-organism. The object of this second investigation is to widen the field of study of rheumatoid affections, reversing, as it were, the usual method of inquiry. The clinician seeks among this heterogeneous group to find some one clear path. We have started along one clear path (the study of the diplococcns) and endeavoured to find our way some little distance into this heterogeneous group.

Taking first the tonsils in rheumatic fever we need only mention the great frequency of tonsillitis in this disease, and the demonstration by Dr. William Hill ${ }^{15}$ some 20 years ago of deep-seated foci of disease in enlarged tonsils removed from the rheumatic. In 1900 we showed that a micrococcus found in rheumatic lesions could be isolated from acute rheumatic angina, and be demonstrated in the tonsils, and further would produce similar lesions. In the next year Fritz Meyer published an extensive paper upon the same subject, and we have returned to the question again to work out Mr. Waugh's suggestion. Mr. Wangh advocates enucleation of the large unhealtby tonsils that are so often present in rheumatic children, and in some carefully chosen cases has done this for us between the rheumatic attacks. These tonsils were seared on" removal, cultures were taken from their depths, and the bistological appearances of them investigated. One example will suffice to show the kind of case investigated, six cases of which have been examined up to the present date.

The patient, a male aged 10 zears and 5 morths, came to the Hospital for Sick Children, Great Ormond-street, in February, 1908 suffering from slight chorea and rheumatic pains. In 1906 he had an attack of rheumatic fever, since whic his usual health, and had always had shortness of breath on exertion. The heart was obviously affected. There was considerable mitra regurgitation, with hypertrophy and dilatation. In addition he had two very large tonsils and was subject to sore-throats. The cervical glands showed a moderate enlargement. In April, when quite recovered from his active rheumatism, Mr. Waugh enucleated both tonsils. Immediatelv after removal the surface was seared, and cultures were taken from the centre of one gland. Histological examination by section and films of the other tonsil showed the presence of streptocoeci in the deep part of the gland. The culture gave an almost pure growth of strepto-diplococci, from which it was easy to isolate this organism in pure culture. Five minims of this cultur injected intravenously into a rabbit produced no result. Two cubie oentimetres produced arthritis and fatal aortic endocarditis. One cubie centimetre in another experiment produced arthritis of the left knee, which slowly recovered. Subsequently smaller doses produced arthritio of the right knee-joint and fatal endocarditis.

We have here but a repetition of the results obtained in our original case in 1899, and independently confirmed by Frit Meyer ${ }^{36}$ in 1901. The particular point of interest lies in the fact that here we are dealing with tonsils in the rheumatic when they are not in the stage of acute inflammation, during an attack of acute rheumatism, but in the latent pericd after previous attacks of the disease.

There can be very little doubt, we believe, that these large unhealthy tonsils are a constant menace to the rheumatic, and that these investigations originated by $\mathbf{M r}$. George Waugh show decisively that there abound in the depths of these disordered tissues strepto-diplococci which will produce with much constancy in appropriate dosage endocarditis, pericarditis, and arthritis on intravenous injection into

15 Tonsillitis in Rheumatic States, 1889

16 A paper read before the Congress of Internal Medicin, Berlin, 1901. 
rabbits. We believe they may well explain some rheumatic relapses. The relation of acute rheumatism and tonsillitis to the diplococcal infection is now so well-defined by clinical observation, histological investigation, and experimental research that it constitutes one of the most satisfactory advances in the study of the disease. We have only to contrast the explanation it gives us with the older nervous and lactic acid theories to realise this advance, which is one, not only of scientific interest, but also of practical importance. We have not investigated other pathways of infection, but great attention has been directed to the importance of unhealthy condition of the teeth. It would be wrong to deny, too, the possibility of infection from the alimentary canal, but we do not think that at present the wide acceptance of auto-infection from the intestines in arthritic diseases has as yet been completely justified by the evidence that has been forthcoming, however probable it may seem on clinical grounds.

When the position of the tonsils is borne in mind it may seem a startling assertion to make that strepto-diplococci may be obtained in pure culture from the depths of the diseased glands, yet it is true, and has happened not only in our experience but in one case was quite independently ascertained by Dr. Grabam Forbes, who had examined a culture from the same case.

We are not pretending that a pure culture is a constant result, but we have invariably found that the streptodiplococci predominated. Again, we do not deny that the same results, both cultural and experimental, might obtain if diseased tonsils from the non-rheumatic were treated in the same way. We have not worked at this point. Should similar results obtain it would not negative the interest of the facts before us, for it is quite evident from our clinical knowledge that there must be a tendency to develop rheumatism, and we have never believed that the exciting cause was not present in the normal throat. As with tuberculosis and pneumococcal infections, so with rheumatism, there are many factors to be considered in addition to the presence of a bacterium.

\section{Investigations rpon Arthritis.}

When the diplococcus of rheumatism is injected intravenously it produces arthritis with great frequency; and further we have convinced ourselves that it may produce it in various forms. In $1902^{17}$ we published with drawings in the Transactions of the Pathological Society a short paper upon the production of an osteo-arthritis-non-suppurative in character-by the intravenous inoculation of a micrococcus isolated from a case of human osteo-arthritis in which death had occurred from misadventure. We believe this to have been the first recorded example of experimental osteoarthritis by intravenous inoculation. Since that date we have produced osteo-arthritis and peri-articular arthritis with the diplococcus rheumaticus, and at the present time we have a very striking specimen of an arthritis resulting from this infection. This occurred in a rabbit which had developed inflammation first of one knee-joint and then of the other, from which it gradually recovered, but 18 months later, though in good health, it limped to some extent upon the hind limbs. This specimen showed that there was a clear fluid in both articulations, but the right patella was dislocated and had formed for itself a new facet on the inner side of the internal femoral condyle and the original facet had lost much of its cartilage, and the bones had been eroded. The left joint showed erosion of cartilage. The occurrence of dislocations of joint surfaces in human "rheumatoid arthritis" is well recognised, and the production of such a deformity is strictly analogous to the condition in the specimen just described, in that there are in both a stretching and damaging of the capsules of the articulations by a non-suppurative process, a damage to the articulating surfaces themselves, and a faulty pull of tendons connected with the joints. At the British Medical Association meeting at Manchester we also demonstrated convincing examples of the "guttering" of osteoarthritis, and published illustrations of these changes in a paper in the Medioal Press and Curcular. ${ }^{18}$ In this paper we also showed that perivascular fibrosis occurred in the capsules of articulations, the subject of experimental and pathological chronic arthritis, explaining therehy the "withering" of joints in rheumatoid affections. We have also produced that rarefying osteitis of bone ends in the neighbourhood of joints affected by chronic disease which is well recognised in " rheumatoid arthritis." Although so far as we can judge from modern writings and discussions the results that we have already published have aroused but little interest we cannot help thinking that the fart that a micro-organism can, on intravenous inoculation, produce all these various forms of arthritic change must in the course of time persuade many to look upon "rheumatoid affections" in a more tolerant spirit. At present the statements that meet one are somewhat of this nature. "Rheumatoid arthritis" is quite different from rheumatic arthritis ; or put as an interrogative, Do you suppose that all "rheumatoid arthritis" is rheumatic?

Investigations of this nature do not pretend to settle such problems, but they do warn us not to classify " rheumatoid affections " by the character of the arthritic lesions, and not to persist in stating dogmatically that acute rheumatism produces a transient arthritis. For ourselves, we believe that just as malignant rheumatic endocarditis is probably a more frequent occurrence than is generally believed, so also, too, is malignant rheumatic arthritis.

We will not again labour here the obstructing influence that we believe the view that salicylate of soda is specific exerts upon advances in the study of rheumatism.

Two further clinical points of minor importance from the scientific view have engaged the attention of one of us. The first is the age at which a rheumatic infection may occur. It seemed to us remarkable that it should delay its appearance to the fifth year and later, for there seemed to us nothing unusual about the general characters of the infection that demanded such a strange limitation. In a paper published in $1908^{10}$ it was shown that rheumatism is by no means rare in the very young, although infancy, being protected by its helplessness from exposure, is to a great extent immune. Thus any difficulty upon the score of age in regarding rheumatism as an infection is groundless.

The second point was an investigation into the afterhistory of scarlatinal rheumatism in a paper published in 1909.20 It was shown that most, and possibly all, of these cases are examples of true rheumatism, probably modified to some extent by the mysterious condition we call scarlet fever.

In conclusion, we would repeat that no explanation of acute rheumatism can, we believe, compare with that which attributes it to an infection with a diplococcus of the streptococcal group; and that although during the last ten years this view has gained but little headway in this country the Chelsea Clinical Society in 1900 marked a forward step in London when they opened a discussion on "Acute Infective Rheumatism."

\section{TWO CASES OF NEPHRECTOMY. ${ }^{1}$}

\section{BY R. ATKINSON STONEY, F.R.C.S. IREL.,}

VISITING SURGEON TO THE ROYAL GITY OF DUBIIN HOSPITAT.

THE following two cases of nephrectomy are, I think, of sufficient interest to merit recording.

CASE 1. - The patient, a man, aged 28 years, while riding a bicycle down-hill on August 2nd, 1909, collided with a man and was thrown off his bicycle and fell on his right shoulder. He fell clear of his machine, and was not conscious of striking the ground except with his shoulder; he got up immediately, but fell down again and was unconscious for about ten minutes. He then went home, and half-an-hour after the accident he vomited. For the next week he remained very quietly in bed, suffering great pain under the ribs on the right side. The pain subsided somewhat, and the next week the patient was able to sit up in bed, and by the end of the week he was up and about, though he did not return to work. On the $19 \mathrm{th}$ - that is, the seventeenth day after the accident-he for the first time noticed blood in his urine. He brought a specimen to hospital with him, and as it was found to contain a large quantity of blood he was at once admitted to the Royal City of Dublin Hospital. The patient was quite positive that this was the first time since the

$$
\text { 19 Quarterly Journal of Medicine, vol. i., No. } 3 .
$$

1 A paper read at the Surgical Section of the Royal Academy of Medicine in Ireland on March 11th, 1910. 